\title{
Modelación matemática a partir de problemas de enunciados verbales: un estudio de caso con profesores de matemáticas
}

Modélisation mathématique à partir de problèmes d'énoncés verbaux: une étude de cas avec les professeurs de mathématiques

Modelagem matemática a partir de problemas de enunciados verbais: estudo de caso com professores de matemáticas

Fecha de recepción: 23 DE MARZO DE 2013/Fecha de aceptación: 6 DE MAYO DE 2015/Fecha de disponibilidad en línea: 30 DE OCTUBRE DE 2015

Encuentre este artículo en http://magisinvestigacioneducacion.javeriana.edu.co/

\section{Resumen}

jhony.villa@udea.edu.co

\begin{abstract}
La literatura internacional sobre educación matemática ha reportado la necesidad de indagar por los aspectos que intervienen en la manera como se desarrolla la modelación matemática en las aulas de clase. En este artículo de investigación se reportan algunos resultados de un estudio de caso cualitativo que indagó por las maneras en que un conjunto de profesores de educación secundaria usa la modelación en la enseñanza de las matemáticas. A partir de observaciones directas, entrevistas, cuestionarios y estudio de episodios fue posible identificar algunos de los argumentos que tienen los profesores para favorecer los problemas de enunciados verbales como principal manera de hacer modelación matemática; estos resultados sugieren la necesidad de generar estrategias que permitan un empoderamiento de los profesores sobre la modelación que trascienda los enunciados verbales estereotipados hacia situaciones más realistas que involucren aspectos de la cotidianidad y la cultura en las matemáticas escolares.
\end{abstract}

\section{Palabras clave}

Modelo matemático; formación de docentes de secundaria; modelización

\section{Transferencia a la práctica}

Este artículo presenta argumentos sobre la necesidad de que en el aula de clase se trascienda el uso problemas de enunciados verbales-rutinarios como la única manera de hacer modelación matemáticas en el aula de clase y, en consecuencia con ello, deriva algunas implicaciones para la formación de profesores que propicien ambientes en los cuales otras relaciones entre las matemática y la "realidad" puedan ser develadas. Lo anterior sugiere que los programas de formación de profesores deber tener en cuenta conocimientos y creencias de los profesores sobre las matemáticas, su enseñanza así como sus consideraciones e ideas sobre la modelación matemática y los factores asociados a ella.

Para citar este artículo / To cite this article / Pour citer cet article / Para citar este artigo

Villa-Ochoa, J. A. (2015). Modelación matemática a partir de problemas de enunciados verbales: un estudio de caso con profesores de matemáticas. magis, Revista Internacional de Investigación en Educación, 8(16), 133-148. http://dx.doi.org/10.11144/Javeriana.m8-16.mmpe 


\section{Keywords}

Mathematical model; secondary teacher development; modeling

\section{Abstract}

International literature on mathematics education has reported the need to investigate the aspects that intervene in the way mathematical modeling takes place in the classroom. This paper presents some results of a qualitative case study that investigated the ways in which a group of high school teachers use modeling when teaching mathematics. From direct observations, interviews, questionnaires and study of events it was possible to identify some arguments that teachers have in order to promote verbal-stated problems as the main way to do mathematical modeling. These results suggest the need to develop strategies that enable an empowerment of teachers on modeling, which transcend the stereotyped verbal-statements towards more realistic situations that involve aspects of daily life and culture in school mathematics.

\section{Transfer to practice}

This article presents arguments about the need of trascending the use of verbal-stated problems as the only way to do mathematical modeling in the classroom. Consequently, it derives some implications for teacher development programs in order to promote environments in which other relations between mathematics and "reality" could be unveiled. This suggests that teacher development programs should consider teacher's knowledge, beliefs about mathematics and their teaching. Also, their considerations and ideas regarding mathematical modeling, and the factors associated with it.

\section{Mots clés}

Modèle mathématique; formation d'enseignants de secondaire; modélisation

\section{Résumé}

La littérature internationale sur l'éducation mathématique a montré le besoin d'étudier la manière comment on développe la modélisation mathématique dans les salles de classe. Dans cet article de recherche on reporte quelques résultats d'une étude de cas qualitative qui a étudié les manières d'utiliser la modélisation mathématique d'un ensemble de professeurs d'éducation secondaire. A partir des observations directes, interviews, questionnaires et l'étude d'épisodes nous a été possible d'identifier quelques-uns des arguments qui ont les professeurs pour favoriser les problèmes d'énoncés verbaux en tant que principal manière de faire la modélisation mathématique; ces résultats montrent le besoin de créer des stratégies qui permettent un renforcement des professeurs par rapport à la modélisation mathématique qui surpasse les énoncés verbaux stéréotypes et se dirige vers les situations plus réels qui impliquent les aspects de la quotidienneté et la culture dans les mathématiques scolaires.

\section{Transfert à la pratique}

Cet article présente les arguments sur le besoin de surpasser l'usage de problèmes d'énoncés verbaux habituels dans la salle de classe en tant que la seule manière de faire la modélisation mathématique et en conséquence avec cela dérivent quelques implications pour la formation de professeurs où on crée des environnements dans les quels d'autres rapports entre les mathématiques et la réalité puissent être montrés. Cela dit que les programmes de formation de professeurs doivent penser dans les connaissances et croyances de professeurs par rapport à la mathématique, leur enseignement ainsi que leurs considérations et idées sur la modélisation mathématique et les facteurs associés à celle-ci.

\section{Palavras-chave}

Enunciados verbais rotineiros; modelagem matemática escolar; formação de professores; situações de modelagem matemática

\section{Resumo}

A literatura internacional sobre educação matemática relatou a necessidade de investigar os aspectos que intervêm na maneira como é desenvolvida a modelação matemática nas salas de aula. Neste artigo de pesquisa são relatados alguns resultados de um estudo de caso qualitativo que investigou as maneiras em que um conjunto de professores de educação secundaria usa a modelagem no ensino das matemáticas. A partir de observações diretas, entrevistas, questionários e estudo de episódios foi possível identificar alguns dos argumentos que os professores têm para favorecer os problemas de enunciados verbais como principal maneira de fazer modelagem matemática; estes resultados sugerem a necessidade de gerar estratégias que permitam um empoderamento dos professores sobre a modelação que transcenda os enunciados verbais estereotipados para situações mais realistas que envolvam aspectos do quotidiano e a cultura nas matemáticas escolares.

\section{Transferência à prática}

Este artigo apresenta argumentos sobre a necessidade de nas aulas transcender o uso problemas de enunciados verbais-rotineiros como única maneira de fazer modelação matemática na aula e, em consequência, deriva algumas implicações para a formação de professores onde forem propiciados ambientes nos quais outras relações entre matemáticas e "realidade" podem ser desvendadas. O dito sugere que os programas de formação de professores precisam levar em conta conhecimentos e crenças dos professores sobre as matemáticas, seu ensino, assim como suas considerações e ideias sobre a modelagem matemática e os fatores associados com ela. 


\section{Introducción}

Uno de los argumentos por los que se recomienda la implementación de la modelación matemática' como recurso en las aulas escolares es el hecho de que, a partir de ella, las matemáticas pueden observarse como una herramienta que permite describir y analizar algunos fenómenos o problemas del entorno en el cual se desenvuelven los individuos cotidianamente. Investigadores como José Joaquín García-García y Edilma RenteríaRodríguez (2013), Rodney Carlos Bassanezi (2002) y Werner Blum y Rita Borromeo-Ferri (2009) argumentan el uso de la modelación y de los modelos en el aula porque ambos posibilitan en los estudiantes el desarrollo de competencias críticas, la comprensión de sus propios contextos, y aspectos sociales como la formación de ciudadanos responsables para participar de los desarrollos de una sociedad que requiere cada vez mayores competencias en modelación.

Argumentos semejantes a los anteriormente presentados sirvieron como base para que el Ministerio de Educación Nacional de Colombia propusiera la modelación matemática como uno de los cinco procesos generales que deben articular los currículos de matemáticas de la Educación Básica y Media (Colombia, MEN, 1998, 2006). Una mirada a tal propuesta permite observar el grado de coherencia entre los propósitos declarados por las regulaciones colombianas para la formación en matemáticas y los resultados de investigación que muestran la modelación matemática como un ambiente prominente para atender a estos propósitos. A pesar de ello, algunos estudios han reportado que tales regulaciones no han sido atendidas por los profesores en sus aulas de clase (Agudelo-Valderrama, 2006) y que, en el caso de la modelación matemática, los mecanismos generados parecen ser insuficientes para que se logre una apropiación e implementación de este proceso en las aulas de clase (Villa-Ochoa \& Ruiz-Vahos, 2009).

La poca implementación de la modelación matemática en el cotidiano escolar ha sido un fenómeno que ha ocupado un lugar en las agendas de investigación internacional. Por ejemplo, Lieven Verschaffel, Erik de Corte e I. Borghart (1997) y Gabriele Kaiser y Katja Maaß (2007) han presentado evidencias de cómo las creencias y concepciones sobre las matemáticas y su enseñanza se convierten en un elemento que interviene en las maneras en que los profesores implementan o no la modelación matemática en la enseñanza. En el mismo sentido, Mogens Niss (2001) apunta que la implementación de la modelación matemática en el cotidiano de las aulas de clase es escasa, debido a que se presentan barreras de diversa naturaleza; entre ellas, las altas demandas matemáticas, pedagógicas y personales que la modelación impone a los profesores.

Los planteamientos expuestos anteriormente ponen de relieve la necesidad de proponer mayores alternativas para establecer vínculos entre las matemáticas escolares y la vida cotidiana de los estudiantes; asimismo la indagación sobre los factores que pueden intervenir en las maneras en que los profesores establecen estos vínculos y los integran en los currículos escolares también debe seguir siendo objeto de estudio.
Descripción del artículo | Article description | Description de l'article | Artigo descrição

Este artículo de investigación es derivado del proyecto El proceso de modelación en las matemáticas escolares del suroeste antioqueño, en el cual se indagó por las maneras en que un conjunto de profesores implementa la modelación matemática como una estrategia en la enseñanza de las matemáticas. El proyecto fue financiado por el Comité para el Desarrollo de la Investigación (CODI) de la Universidad de Antioquia y la Dirección de Regionalización.

1 En términos de Jhony Alexander Villa-Ochoa y Mario de Jesús Berrío (2015), la modelación matemática en educación matemática o modelación matemática escolar puede entenderse como el proceso de estudio de situaciones, fenómenos o problemas propios de los contextos cotidianos o de otras ciencias. Este estudio involucra la producción o uso de modelos matemáticos. 
Un argumento adicional que fundamenta los anteriores requerimientos investigativos puede observarse en las ideas de Lieven Verschaffel, Wim van Dooren, Brian Greer y Swapna Mukhopadhyay (2010), quienes puntualizan que, aunque los señalamientos anteriormente presentados por Mogens Niss pueden llevar a una limitada implementación de la modelación en las aulas de clase, aún es necesario poner atención a cómo los profesores regulares piensan sobre la modelación, cómo la implementan y establecen conexiones entre las matemáticas escolares y la realidad; asimismo, cómo ellos determinan si los estudiantes deben estar expuestos a experiencias realistas de modelación y cómo y cuándo deben hacerlo.

Las afirmaciones de Lieven Verschaffel, Wim van Dooren, Brian Greer y Swapna Mukhopadhyay (2010) ponen de manifiesto la necesidad de indagar por las consideraciones que los profesores tienen sobre las matemáticas escolares y el papel que cumple la modelación frente a ellas mismas; de igual forma, es necesario resaltar la importancia de investigar las maneras en que los profesores pueden implementar "procesos de modelación" en el aula de clase y, de esta manera, obtener información que permita comprender el papel que los contextos propios de los estudiantes juegan en las matemáticas escolares. Así, la investigación, de la cual se desprende este artículo, indagó por las consideraciones que un conjunto de profesores tenía sobre las situaciones que usaban en el aula de clase y con las cuales justificaban la implementación de procesos de modelación matemática; de ese modo, fue posible reconocer algunos de los elementos que parecen intervenir en tal implementación.

\section{Antecedentes teóricos}

Conforme se mencionó en el apartado anterior, la literatura internacional ha reportado que las creencias y concepciones que tienen los profesores sobre las matemáticas y su enseñanza se convierten en un aspecto que se involucra en su forma de valorar el papel de la modelación en la enseñanza y el aprendizaje de las matemáticas. De manera particular, Lieven Verschaffel, Erik de Corte e I. Borghart (1997) presentaron un estudio en el que analizaron las concepciones y creencias de futuros profesores de matemáticas sobre el papel que tiene el conocimiento del mundo real concerniente al contexto de un problema en la interpretación y la solución de enunciados verbales aritméticos (Word problems). En particular, el estudio observó: (1) las respuestas espontáneas de los futuros profesores a un conjunto de enunciados verbales con supuestos para la modelación de problemáticas y (2) las evaluaciones que estos futuros profesores hacían de las respuestas de los alumnos frente a si toman o no en cuenta los aspectos relevantes del conocimiento de mundo real.

La investigación de Lieven Verschaffel, Erik de Corte e I. Borghart (1997) proporciona evidencias que enfatizan que, al igual que otras investigaciones realizadas con niños, hay una tendencia fuerte y resistente entre los futuros docentes para excluir conocimiento del mundo real y las consideraciones realistas cuando se trata de problemas verbales aritméticos en las tareas de enseñanza. De igual manera, los resultados muestran que los participantes de su investigación tienen una creencia, según la cual atribuyen poca importancia a los problemas no estructurados y no estándar; e incluso, consideran que tales problemas pueden ser perjudiciales para la enseñanza de la aritmética en la escuela primaria, puesto que conducen a que los niños se alejen del aprendizaje de las maneras de encontrar respuestas numéricas correctas a estos problemas por medio de las operaciones aritméticas ocultas en el enunciado. 
De otro modo, Gabriele Kaiser y Katja Maaß (2007) analizaron las creencias de un conjunto de profesores alemanes sobre las matemáticas y su enseñanza. Los resultados del estudio de estas investigadoras muestran que las creencias formalistas sobre la naturaleza de las matemáticas (conjunto de reglas lógicas y de fórmulas) y sobre su enseñanza (fines de formación predominantemente esquemáticos) se muestran como aspectos centrales que alimentan la brecha existente entre la relevancia de la modelación matemática (evidenciada en la investigación) y su menor importancia en el día a día de las aulas de clase. Para las autoras, las creencias de los profesores sobre las matemáticas y su enseñanza pueden ser observadas como una razón fundamental en la poca implementación de la modelación y las aplicaciones en las aulas de clase.

En contraste con los trabajos de Lieven Verschaffel, Erik de Corte e I. Borghart (1997), Cinzia Bonotto (2007) sugiere la necesidad de reemplazar los problemas estereotipados por enunciados más realistas que enfaticen mayores vínculos entre las matemáticas y el conocimiento extraescolar. Para ello, la autora sugiere (i) cambiar el tipo de actividad destinada a crear interacción entre el mundo real y las matemáticas hacia situaciones problemáticas más realistas y menos estereotipadas, (ii) cambiar las concepciones de los estudiantes, sus creencias y actitudes hacia las matemáticas (incluso de los profesores), y (iii) el cambio de cultura escolar mediante la creación de nuevas normas sociomatemáticas. Para la autora, los artefactos culturales son relevantes para los estudiantes porque hacen parte de su experiencia de la vida real, es decir, ofrecen importantes referencias a situaciones concretas; ello les permite mantener sus procesos de razonamiento y estar atentos a revisar sus inferencias. Para alcanzar este tipo de ambientes de clase, Cinzia Bonotto propone una serie de retos para los profesores de matemáticas, algunos de ellos relacionados con el trabajo de Gabriele Kaiser y Katja Maaß (2007) en cuanto a las creencias sobre el papel del conocimiento cotidiano en la enseñanza de las matemáticas; pero en otras, sugiere que el profesor debe modificar su actitud hacia las matemáticas (más allá de las influencias de cómo las aprendió) y concebir las relaciones entre estas y el mundo real como punto de partida para las actividades en el aula; de igual manera, sugiere que el profesor debe investigar las ideas matemáticas y las prácticas de las comunidades culturales, étnicas, lingüísticas de sus estudiantes y tenerlas en cuenta para el trabajo matemático en el aula de clase.

\section{El estudio}

Este artículo reporta parte de una investigación en la que se involucraron cuatro profesores que se desempeñaban en diferentes niveles de instituciones educativas estatales. Los cuatro participantes habían sido formados como profesores de matemáticas y, adicionalmente, uno de ellos tenía una especialización en enseñanza de las matemáticas. Los cuatro profesores aceptaron voluntariamente participar de esta investigación después de haberse desempeñado como cooperadores de un proceso de práctica docente de cuatro estudiantes (futuros profesores) de un programa de licenciatura en educación con énfasis en matemáticas. Los profesores fueron observados en su ejercicio docente por los investigadores y posteriormente se les aplicó un cuestionario; luego se desarrolló una sesión grupal de cinco horas en la cual se discutió acerca de los episodios preparados sobre la modelación y, finalmente, se les hizo una entrevista.

Los episodios que direccionaron el desarrollo de la sesión grupal se describen a continuación:

Episodio 1. La caricatura. Se trata de una historieta en la cual se presentan cuatro escenas que describen la manera en que un profesor ofrece respuestas a la pregunta ipara qué sirve la matemática? Ante el cuestionamiento de los estudiantes, el profesor ofrece algunas formas de establecer relaciones entre las matemáticas y la "realidad". Tal historieta puede encontrarse en los trabajos de Jhony Alexander Villa-Ochoa y Carlos Mario Jaramillo-López (2011) y de Jhony Alexander Villa-Ochoa, Carlos Alberto Bustamante-Quintero, Mario de Jesús BerríoArboleda, Jesús Aníbal Osorio-Castaño y Diego Alexander Ocampo-Bedoya (2009).

Episodio 2. Este episodio corresponde a la descripción teórica que se destina a la modelación matemática en el documento Lineamientos curriculares: matemáticas (Colombia, MEN, 1998). En este documento se describe el siguiente enunciado como una manera de ilustrar al lector formas de situaciones de modelación: "Una familia de cuatro (4) personas ha invitado a tres (3) amigos a comer a su casa. ¿Cuántos puestos se pondrán en la mesa?" (Colombia, MEN, 1998, p. 78).

Episodio 3. El problema de consumo de internet. Se trata de una situación en la que a partir de la pregunta: ¿Cuál de las propuestas de consumo de internet existentes en el mercado será la más conveniente para las diversas necesidades de los compañeros del grupo? Se muestra una serie de momentos por medio de los cuales podría atravesar el proceso de modelación en las aulas de clase (Villa-Ochoa, Bustamante-Quintero, Berrío-Arboleda, Osorio-Castaño \& Ocampo-Bedoya, 2009).

El método adoptado para esta investigación fue el estudio de casos, dado que se prestó especial atención a las formas en que los profesores reconocen la modelación en el aula de clase y cómo se lleva a cabo en las aulas escolares. 
Este estudio se interesó por las consideraciones que los profesores tienen por la modelación matemática; en ese sentido, se puso especial atención a las verbalizaciones de los profesores en sus diálogos con los colegas, con los investigadores y con sus estudiantes en el aula de clase; los registros escritos que produjeron también se convirtieron en objeto de análisis. Los datos recogidos fueron registrados por diferentes medios (audio, video, diarios de campo y otros registros escritos) para posteriormente organizarlos y analizarlos. Se usaron diferentes recursos de evidencia ya que, de acuerdo con Robert K. Yin (2009), la ventaja más importante de hacer esto es la posibilidad de desarrollar una línea convergente de indagación y procesos de triangulación y de corroboración.

Para el análisis, el material audiovisual fue revisado, transcrito y codificado por cada uno de los investigadores; también se digitalizaron y codificaron los diferentes registros escritos producidos por los participantes. Cada integrante del equipo investigador hizo un primer análisis de los datos, posteriormente se triangularon con los análisis del equipo en conjunto.

En la tabla 1 se presentan otros detalles de los instrumentos y algunos resultados de la investigación.

Tabla 1

Otros detalles de los instrumentos y resultados de la investigación

\begin{tabular}{|c|c|c|c|c|}
\hline Instrumentos & Registro & Ejecución & Propósito & Logros \\
\hline $\begin{array}{l}\text { Observaciones } \\
\text { en el aula de } \\
\text { clase }\end{array}$ & $\begin{array}{l}\text { Diarios de } \\
\text { campo }\end{array}$ & $\begin{array}{l}\text { Abril a } \\
\text { junio de } \\
2008\end{array}$ & $\begin{array}{l}\text { Identificar las principales } \\
\text { tendencias, metodologías, } \\
\text { actividades y tareas que } \\
\text { utilizan los profesores } \\
\text { en el desarrollo de sus } \\
\text { sesiones de clase. }\end{array}$ & $\begin{array}{l}\text { Se pudo determinar que los profesores } \\
\text { privilegian estrategias expositivas en las clases } \\
\text { de matemáticas. Estas estrategias siguen } \\
\text { generalmente la secuencia: Introducción } \rightarrow \\
\text { Presentación o definición matemática del } \\
\text { tema } \rightarrow \text { Explicación } \rightarrow \text { Ejemplos } \rightarrow \text { Ejercicios } \\
\text { y/o Aplicaciones } \rightarrow \text { Evaluación. }\end{array}$ \\
\hline $\begin{array}{l}\text { Estudio de epi- } \\
\text { sodios }\end{array}$ & $\begin{array}{l}\text { Audio y } \\
\text { video }\end{array}$ & $\begin{array}{l}\text { Sesión de } \\
5 \text { horas en } \\
\text { la segunda } \\
\text { semana de } \\
\text { noviembre } \\
\text { de } 2008\end{array}$ & $\begin{array}{l}\text { Identificar algunas } \\
\text { creencias acerca } \\
\text { de las matemáticas } \\
\text { escolares y contribuir } \\
\text { a la construcción de } \\
\text { reflexiones por parte de } \\
\text { los profesores; reconocer } \\
\text { las interpretaciones sobre } \\
\text { diversas situaciones de } \\
\text { modelación. }\end{array}$ & $\begin{array}{l}\text { Se construyeron reflexiones acerca del } \\
\text { papel de las matemáticas en la solución } \\
\text { de problemas del "mundo real". Además, } \\
\text { la necesidad de desarrollar un "sentido de } \\
\text { realidad" que posibilite establecer vínculos } \\
\text { entre el "mundo real" en el contexto escolar y } \\
\text { las matemáticas escolares. }\end{array}$ \\
\hline Cuestionario & Escrito & $\begin{array}{l}\text { Una hora } \\
\text { en la } \\
\text { primera } \\
\text { semana de } \\
\text { diciembre } \\
\text { de } 2008\end{array}$ & $\begin{array}{l}\text { Recolectar información } \\
\text { sobre el porqué y el cómo } \\
\text { de las matemáticas en la } \\
\text { educación secundaria ( } 11 \\
\text { a } 15 \text { años) e identificar } \\
\text { algunas creencias acerca } \\
\text { de las matemáticas } \\
\text { escolares. }\end{array}$ & $\begin{array}{l}\text { Se pudo determinar que los tres tipos de } \\
\text { situaciones usadas en el aula de clase: } \\
\text { (1) Situaciones o ejercicios que favorecen las } \\
\text { realidades inventadas (Alsina, 2007), } \\
\text { (2) ejercicios prototipo al interior de las } \\
\text { matemáticas, y } \\
\text { (3) ejercicios de aplicación de los temas en } \\
\text { situaciones en contexto. }\end{array}$ \\
\hline Entrevista & $\begin{array}{l}\text { Audio y } \\
\text { video }\end{array}$ & $\begin{array}{l}\text { Segunda } \\
\text { semana de } \\
\text { febrero de } \\
2009\end{array}$ & $\begin{array}{l}\text { Recolectar información } \\
\text { sobre las creencias y } \\
\text { sobre el porqué de los } \\
\text { problemas reales en las } \\
\text { matemáticas escolares. }\end{array}$ & $\begin{array}{l}\text { Se pudo observar la existencia de al menos } \\
\text { tres creencias: } \\
\text { (1) las matemáticas primero seenseñan y luego } \\
\text { se aplican, } \\
\text { (2) la construcción de un modelo es cuestión } \\
\text { de construir una representación de la situa- } \\
\text { ción, y } \\
\text { (3) un problema real es cualquier situación } \\
\text { que utilice palabras de uso cotidiano y que } \\
\text { sirva para ver la aplicación de las matemá- } \\
\text { ticas al mundo real. }\end{array}$ \\
\hline
\end{tabular}

Fuente: elaboración propia 


\section{Resultados}

Este artículo se fundamenta en el análisis de los datos recolectados en tres de los cuatro profesores, los cuales se denominan con los seudónimos de Alexander, Jesús y José. Los tres profesores fueron escogidos porque en ellos se observaron consideraciones que parecen condicionar las maneras en que usan situaciones denominadas por ellos como "reales" y por medio de las cuales justifican la implementación de la modelación matemática en sus aulas de clase. Los resultados correspondientes al cuarto profesor pueden encontrarse en los trabajos de Jhony Alexander
Villa-Ochoa y Carlos Mario Jaramillo-López (2011) y de Jhony Alexander Villa-Ochoa, Carlos Alberto Bustamante-Quintero, Mario de Jesús Berrío-Arboleda, Jesús Aníbal Osorio-Castaño y Diego Alexander Ocampo-Bedoya (2009).

Para Alexander, Jesús y José, las matemáticas son una "poderosa" herramienta para entender diversas situaciones de la "realidad". Ellos se apoyan en esta aserción para justificar la necesidad de proporcionar situaciones que establezcan conexiones entre las matemáticas enseñadas y las situaciones "reales".

En la tabla 2 se muestran algunas de las evidencias recopiladas en el proceso investigativo.

Tabla 2

Algunas evidencias del trabajo investigativo

\begin{tabular}{|c|c|c|}
\hline Profesor & Verbalizaciones [análisis de episodios] & $\begin{array}{l}\text { Acciones en el aula de clase } \\
\text { [observaciones en clase] }\end{array}$ \\
\hline 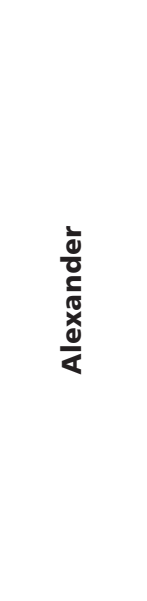 & $\begin{array}{l}\text { Frente al episodio 3, el profesor dijo: "Son situaciones } \\
\text { reales que a los estudiantes les puede gustar, pero el } \\
\text { problema es que ellos dirán que quieren es la fórmula } \\
\text { para resolver las preguntas y no la solucionan". } \\
\text { En el episodio } 2 \text { dijo: "Aunque estas son situaciones } \\
\text { que contienen las matemáticas que se les están } \\
\text { enseñando, no son problemas tan grandes que } \\
\text { los estudiantes no tengan las [herramientas] } \\
\text { matemáticas para hacerlos [solucionarlos]". } \\
\text { En el episodio } 3 \text { dijo: "[...] además, eso quita mucho } \\
\text { tiempo [refiriéndose a la implementación de una } \\
\text { situación presentada en el episodio 3] y siempre el } \\
\text { coordinador está diciendo: 'ipilas!, ipilas!, ¿cómo va } \\
\text { con el desarrollo del plan de área?'". }\end{array}$ & $\begin{array}{l}\text { En las clases predominó un método expositivo en } \\
\text { el cual se presentan las definiciones, se muestran } \\
\text { ejemplos procedimentales y luego algunas } \\
\text { aplicaciones. } \\
\text { En el tema de las ecuaciones, el profesor explicó } \\
\text { primero los métodos de sustitución, igualación } \\
\text { y reducción para resolución de sistemas de } \\
\text { ecuaciones. [A la semana siguiente] Dedicó las } \\
\text { clases para plantear problemas de aplicación } \\
\text { de ecuaciones, explicó primero problemas de } \\
\text { ecuaciones lineales con una incógnita y luego de } \\
\text { sistemas de ecuaciones. En el libro de texto que } \\
\text { seguía con los estudiantes había un proyecto de } \\
\text { conexión con otras ciencias; el profesor mencionó } \\
\text { a los estudiantes que los que pudieran lo hicieran } \\
\text { volutariamente, pero lo dejó para trabajo opcional } \\
\text { de los estudiantes. }\end{array}$ \\
\hline$\stackrel{n}{\stackrel{n}{y}}$ & $\begin{array}{l}\text { Frente al episodio 2, el profesor dijo: "Es [una } \\
\text { situación] ireal! Ahí los estudiantes pueden trabajar } \\
\text { con las operaciones básicas y así las matemáticas no } \\
\text { son solo operaciones con números en abstracto, sino } \\
\text { que hacen parte de un contexto de ellos mismos. [...] } \\
\text { Mire, profe [refiriéndose al investigador], también } \\
\text { les ayuda a pasar del lenguaje de la realidad al } \\
\text { matemático que eso, en problemas de ecuaciones } \\
\text { o en física, es de lo más difícil para ellos [para los } \\
\text { estudiantes]". }\end{array}$ & $\begin{array}{l}\text { El profesor explicó las condiciones para que haya } \\
\text { proporcionalidad directa, propuso a los estudiantes } \\
\text { un conjunto de magnitudes para que ellos } \\
\text { establecieran las relaciones de proporcionalidad. } \\
\text { Puso luego [a los estudiantes] a realizar el conjunto } \\
\text { de ejercicios que estaban en el libro [de texto] que } \\
\text { seguían los estudiantes. }\end{array}$ \\
\hline 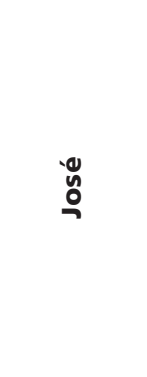 & $\begin{array}{l}\text { Frente al episodio 2, el profesor dijo: "Para mí es real, } \\
\text { yo puedo poner a los estudiantes a que se sienten en } \\
\text { rodela [en forma de circunferencia] o alrededor de una } \\
\text { mesa para que hagan la situación. Desde la primaria } \\
\text { [nivel educativo para estudiantes entre } 6 \text { y } 10 \text { años de } \\
\text { edad] los muchachos [estudiantes] están acostumbra- } \\
\text { dos a que les mostremos las aplicaciones de las ma- } \\
\text { temáticas por medio de problemas de manzanas, ir a } \\
\text { la tienda y comprar, y así, eso es importante para que } \\
\text { ellos sepan que las matemáticas tienen aplicaciones en } \\
\text { la vida [...]". }\end{array}$ & $\begin{array}{l}\text { El profesor comenzó el trabajo con operaciones con } \\
\text { expresiones algebraicas. Explicó a los estudiantes que } \\
\text { era muy importante que ellos sacaran expresiones de } \\
\text { problemas. Por eso, puso una lista de ejercicios para } \\
\text { que los estudiantes las escribieran en forma algebrai- } \\
\text { ca. Por ejemplo: la suma de dos números: } x+y \text {; } \\
\text { suma de dos cuadrados: } x^{2}+y^{2} \text {, una suma al cuadra- } \\
\text { do: }(x+y)^{2} \text {, etc. }\end{array}$ \\
\hline
\end{tabular}

Fuente: elaboración propia

2 magis no corrige las transcripciones de las fuentes primarias de las investigaciones que presenta. 
Según las evidencias presentadas en la tabla 2, Alexander, Jesús y José reconocen la importancia de involucrar situaciones "reales" en la enseñanza de las matemáticas; eso pone a estos profesores en un contexto diferente al reportado por Gabriele Kaiser y Katja Maaß (2007), pues la enseñanza para estos profesores no debe circunscribirse únicamente a los aspectos formales de las matemáticas ni solo a la transmisión de reglas y de su lógica interna. Sin embargo, tampoco se encuentra evidencia que para estos profesores la modelación matemática tenga una responsabilidad en la constitución de las matemáticas mismas y, por tanto, en su enseñanza; contrario a ello, para los profesores participantes de este estudio, los denominados "problemas reales" son una parte secundaria dentro del conocimiento matemático y, como tal, se restringe a la resolución de algunos enunciados que se observan más como una "aplicación" del conocimiento matemático enseñado que en un medio para aprenderlo. En coherencia con lo anterior, estos enunciados parecen reducirse a un mecanismo para evaluar la habilidad de los estudiantes para registrar mediante una expresión simbólica una relación matemática "revestida" en un enunciado verbal.

En los siguientes apartados se realiza un análisis más detallado del tipo de enunciados usados por los profesores y se presentan algunas razones por medio de las cuales ellos justifican su uso como una manera de modelar matemáticamente en el aula de clase.

Los enunciados verbales (rutinarios) como una manera

de abordar la "realidad"

El enunciado verbal del episodio 2 puede considerarse en los trabajos de Lieven Verschaffel y sus colaboradores Wim van Dooren, Brian Greer y Swapna Mukhopadhyay (2010) como un S-item, pues su solución implica la aplicación de unas operaciones aritméticas obvias con los números proporcionados en el enunciado. Según la evidencia presentada en la tabla 2, Alexander asume que este enunciado representa "la realidad"; para ese profesor, este tipo de problemas "reales" parecen ser pertinentes en el aula de clase porque los estudiantes los pueden resolver de manera más inmediata. Según Alexander, los estudiantes pocas veces están motivados a resolver problemas más amplios pues, generalmente, están buscando aplicar un algoritmo para resolverlos. El uso de los problemas presentados como enunciados verbales (por ejemplo, episodio 2) parece ser una estrategia que profesores como Alexander utilizan para encarar la poca disposición de algunos estudiantes frente a tareas menos estructuradas, más complejas. Como evidencia para apoyar esta idea, se presenta el comentario que el profesor hace en el estudio del episodio 1.

Esa pregunta [¿para qué sirven las matemáticas?] la encuentra uno muchas veces. Uno llega y les dice [a los estudiantes] para qué [sirven las matemáticas], incluso les hace esquemas de dónde se utilizan. Sin embargo, el pelao [estudiante], él quiere ver, uhhh, no la parte matemática, sino la parte procedimental, la parte donde se utilice. Pero no quiere aprender los conceptos y creo que algo que hace falta en las matemáticas es aprender conceptos. Yo estudio mecánica automotriz, una tecnología [refiriéndose a un programa de Educación Superior cuya duración aproximada es de 3 años], y nos comentaba un profesor de electrónica que prácticamente la electrónica sería imposible sin las matemáticas; porque todo lo que tiene qué ver con circuitos y con todo, es realmente matemáticas. Entonces uno se pone ahí a observar y se va motivando, pero [a] los niños uno les puede mostrar cómo se hace, cómo llevarlos a la vida real $y$, sin embargo, siguen 
los mismos dos o tres muchachos que siempre están pendientes de todo y los otros [dicen] 'no es que a mí me interesa es el aparato ya, yo no quiero saber para qué se utiliza' entonces esa es la gran dificultad.

Estos elementos muestran que Alexander parece haberse dado cuenta de que las conductas que él menciona en algunos estudiantes no siempre están en correspondencia con una exigencia de las tareas realizadas en las matemáticas escolares y que tales actuaciones representan la construcción de un conjunto de creencias y comportamientos que veneran los buenos desempeños con mínimos esfuerzos (Alan H. Schoenfeld, 1991, citado por Verschaffel, van Dooren, Greer \& Mukhopadhyay, 2010). Ante la falta de estrategias que evidencien maneras de romper con ese conjunto de creencias y comportamientos, los profesores parecen acudir a los enunciados verbales (rutinarios) como una manera de atender a los condicionamientos impuestos por estos comportamientos, y como una forma de poner en correspondencia inmediata (con mínimo esfuerzo) las matemáticas y los supuestos "contextos de los estudiantes".

Si bien la literatura ha mostrado que los enunciados verbales, como los sometidos a discusión en el episodio 2, son generalmente una de las estrategias más usadas por los profesores en sus aulas de clase, también es cierto que se debe crear la necesidad de integrar otras formas de implementar la modelación matemática (i.e., problemas realistas, no estructurados, etc.), de tal manera que los enunciados verbales, considerados como problemas rutinarios, no constituyan estereotipos que buscan simplificar la complejidad de situaciones más realistas. Frente a este tipo de enunciados, Susan Gerofsky (2010), basada en autores posmodernos, argumenta la imposibilidad de abordar la "realidad" por medio de los enunciados verbales (word problems), asimismo discute las nociones de transparencia o realidad autoevidente que parecen estar en algunas de las consideraciones de Alexander.

Otro aspecto que parece alimentar ese conjunto de creencias y comportamientos mencionado anteriormente, tiene relación con ciertas prácticas observadas en los profesores descritos en este estudio. En particular, para estos profesores hay un argumento adicional para restringir el uso de contextos realistas en las matemáticas escolares; este argumento se cimienta en la idea de que los estudiantes deben conocer previamente las matemáticas involucradas en las situaciones que deben abordar y, en consecuencia, se asume que en contextos más amplios podrían encontrarse matemáticas que no conocen; por tanto, no podrían abordarlos. De estas verbalizaciones y de los elementos presentados en la tabla 2, se aprecian mayores evidencias sobre las consideraciones que tienen los profesores implicados frente a los enunciados verbales rutinarios como la herramienta más usada para establecer relaciones entre las matemáticas y los contextos de los estudiantes.

Como se pudo apreciar en la tabla 2, para el caso de Alexander, si bien es cierto que los libros de texto pueden no involucrar contextos de modelación propiamente dichos, también es cierto que en los casos en que presentan proyectos o actividades que imponen cierto conocimiento frente a contextos determinados, el profesor parece omitir su desarrollo en el aula de clase. Para profundizar un poco más en estas observaciones se estableció el siguiente diálogo con Alexander:

Investigador: ¿Por qué son importantes este tipo de problemas [enunciados verbales] en las matemáticas? 
Alexander: $\quad$ Porque permiten ver las matemáticas aplicadas a la cotidianidad, por ejemplo, las matemáticas para las compras, para tomar decisiones, y así [...].

Investigador: Bueno, y ¿qué otros problemas o situaciones o fenómenos se pueden usar?

Alexander: $\quad i A h !$ pues muchos, pero el problema es que los estudiantes no son capaces. Mire que, por ejemplo, para hacer un problema como el de internet [Episodio 3] ellos tienen que haber visto primero funciones, además el problema es que eso se lleva mucho tiempo y los pelaos [estudiantes] quieren son las fórmulas, hacerlo de la forma más fácil.

Investigador:

Y si un problema aparece en un libro del estudiante [texto] como un proyecto para que el estudiante investigue, ¿ves importante que él lo realice?

Alexander:

Claro que es importante que lo haga, aunque como son tan largos yo los dejo para que lo hagan voluntario, [a] ellos generalmente no les gustan esos problemas porque, como le digo, les gustan son los más simples para aplicar fórmula y ya.

Semejante a las consideraciones de Alexander en la tabla 2, José y Jesús también expresaron que los enunciados verbales, como los discutidos en el episodio 2, son pertinentes para realizar "traducciones entre el lenguaje natural y la matemática". En la discusión sobre el episodio 3 (la adquisicion de un plan de internet) se presentó el siguiente diálogo:

$\begin{array}{ll}\text { Investigador: } & \text { Bueno, continuando entonces con la discusión vamos ahora } \\ \text { a hablar de esta actividad [refiriéndose a la situación descrita } \\ \text { en el episodio 3: El problema de consumo de internet] ¿Creen } \\ \text { que es una situacion real? iSe puede adaptar para trabajar } \\ \text { en el aula de clase? } \\ \text { jClaro que es real! Ahí están trabajando con tarifas e internet } \\ \text { Jesús: } \\ \text { adaptar con la cuenta de la luz [refiriéndose a la factura de } \\ \text { energía] o con las empresas de por acá. El problema de eso es } \\ \text { que gasta mucho tiempo, los pelaos muchas veces no llevan } \\ \text { las facturas para trabajar en clase. }\end{array}$

Investigador: José, y ¿usted qué piensa?

Jose:

Pues yo también pienso que es real, pero - como dice Jesús- el problema es que eso no se puede hacer en el salón porque no se alcanza a cumplir el programa, los padres van a estar criticando porque se pierde mucho tiempo y los rectores con mayor razón.

Investigador: Y, ihan intentado hacer situaciones parecidas en clase?

Jesús: $\quad$ No, porque en mi colegio no dejan [...]

Jose: $\quad$ Pues yo los he llevado a la sala de sistemas, he buscado actividades por internet, [...] pero ha sido más bien poco porque hay muchos grupos que quieren ir y la sala no da abasto.

De la evidencia presentada en la tabla 2 y en el anterior diálogo, se puede observar que los profesores ponen de manifiesto ciertas limitaciones impuestas por la manera en que están organizados sus "currículos" como una justificación de su preferencia por usar contextos más realistas y actividades no estructuradas en la modelación matemática. Según los profesores, los currículos se muestran "rígidos" frente a su organización por 
medio de contenidos y en términos del tiempo de la clase. Según estas apreciaciones, la existencia de esos "currículos rígidos" es otra de las razones en las que los profesores se apoyan para justificar el uso de problemas rutinarios enunciados verbalmente.

De acuerdo con los elementos evidenciados hasta el momento, estos profesores parecen tener una mirada sobre la modelacion que se agota en la "traducción del lenguaje natural al lenguaje matemático" y en la "ilustración de ejemplos de conexiones entre las matemáticas y la realidad". En el desarrollo de este estudio no se obtuvo evidencia de la existencia de consideraciones de los profesores que reconocieran la modelación como un medio para que los estudiantes tengan una participación activa en el ámbito social y cultural (Araújo, 2009), o como un ambiente que facilita una estructura pedagógica que promueve producción o conversión de conocimientos de los estudiantes mediante el estudio de ideas matemáticas presentes en realidad (Rosa \& Orey, 2012), entre otras. Quizás el desconocimiento de estos y otros aportes de la modelación matemática puede llevar a estos profesores a una "actitud pasiva" que no rompe con la linealidad impuesta por los currículos ni con ese conjunto de actitudes "facilistas" que, según los profesores, se puede observar en sus estudiantes. Estos elementos imponen otros retos a los profesores (y a los programas de formación de profesores) que les permitan hacer frente a tales situaciones, como se discutirá más adelante en este artículo.

\section{La modelación como la producción de una representación de enunciados verbales rutinarios}

En el apartado anterior se mostraron algunas razones que hacen que los enunciados verbales (rutinarios) sean privilegiados por algunos profesores como una manera de hacer modelación matemática en las aulas de clase. Los hallazgos de este estudio dan cuenta de otros aspectos que se muestran como favorables a este tipo de prácticas; así, por ejemplo, en los participantes de este estudio se observó la presencia de algunas ideas de la modelación asociada a "[...] la construcción de modelos matemáticos a partir de problemas de la realidad", y que "los modelos matemáticos son símbolos [...] u otra representación matemática". En estas dos ideas sobre la modelación matemática están presentes otras como "la producción de representaciones" y "la realidad"; frente a esta última, las verbalizaciones de los profesores en la tabla 2 dan cuenta de que la noción de "realidad" parece asociarse con cualquier tipo de enunciado que, al incluir palabras que describen situaciones hipotéticas o ficticias, adquiere el estatus "real" por el hecho de poder simularlo en el aula.
Para profundizar en las consideraciones de los profesores sobre la modelación, se instó a los participantes a expresar sus ideas frente a la idea de modelo matemático; en este aspecto emergieron aserciones como "expresiones y algoritmos matemáticos" (José), "procedimientos a seguir [...], son como fórmulas algebraicas, gráficos matemáticos" (Alexander). Estas ideas se ratificaron en los profesores cuando discutieron el episodio 2, en el cual se hizo la lectura del apartado de los Lineamientos curriculares: matemáticas (Colombia, MEN, 1998); en ese documento, el término modelo matemático se usa como una de las partes de un determinado ciclo de la modelación, y se afirma que "[un] modelo consta esencialmente de ciertos objetos matemáticos, que corresponden a los 'elementos básicos' de la situación original o del problema formulado, y de ciertas relaciones entre esos objetos, que corresponden también a relaciones entre esos 'elementos básicos'"(p. 98).

Como pudo observarse en las evidencias presentadas anteriormente, en los tres profesores reportados en este artículo parecen converger la idea de "realidad" vinculada a la "posibilidad de imaginar u ocurrir" y la idea de modelación como "la acción de construir una representación de dicha realidad"; la conjunción de estos dos tipos de ideas en los profesores parece abonar el terreno para que los enunciados verbales rutinarios se conviertan en una de las principales maneras de hacer modelación matemática en las aulas escolares.

Valorar los modelos solo como representaciones y limitar la modelación en una definición que pone solo énfasis en la "construcción" de los mismos sin atender a una discusión sobre los contextos, fenómenos y situaciones de los cuales provienen, parece tener algunas implicaciones en la comprensión de la complejidad de la modelación, sus ventajas y limitaciones en el aula de clase; pero, sobre todo, parece limitar la modelación a la "construcción de representaciones" de cualquier tipo de enunciados, incluso de aquellos que claramente son artificiales, no realistas o que evocan ciertas situaciones que podrían imaginarse o simularse en el aula.

Estas consideraciones se ponen en concordancia con los registros en los diarios de campo producidos por los investigadores, a partir de las observaciones en el aula de clase de estos profesores. En un análisis de estos diarios se observa que los profesores José y Alexander hicieron alusión al término "modelo" como una representación usada en la sección de "aplicaciones de la matemática", que ellos desarrollaban después de hacer alguna sesión de explicaciones de cierta temática particular. Tal sesión se centró en ciertas tareas prototipo de los libros de texto o, en algunos casos, producidas por los profesores mismos. 
Una vez más aparecen los enunciados verbales (rutinarios) como una manera de ilustrar las relaciones entre las matemáticas y los "contextos cotidianos" de los estudiantes. Sin embargo, para los profesores analizados en este artículo, estos enunciados se muestran como descripciones textuales prototipo que pueden generar una idea estereotipada de las matemáticas y, sobre todo, una mirada reducida de las relaciones entre ellas y la cultura.

Lieven Verschaffel y sus colaboradores Wim van Dooren, Brian Greer y Swapna Mukhopadhyay (2010) mencionan que desde la literatura internacional puede delimitarse una línea de argumentación que, basada en perspectivas socioculturales, socioconstructivistas e interaccionistas, permite señalar que los estudiantes, después de estar durante mucho tiempo inmersos en una cultura de las matemáticas tradicionales, evidencian una aproximación de la modelación reducida a un simple tratamiento algorítmico que no exige ninguna reflexión seria acerca de las posibles limitaciones de las realidades del contexto, del problema que puede poner en peligro la adecuación de sus modelos y soluciones estándares.

\section{Discusión}

La literatura con frecuencia reporta que los enunciados verbales (rutinarios) son, tradicionalmente, observados como el vehículo para introducir la modelación y las aplicaciones matemáticas en las aulas de clase (Bonotto, 2007; Verschaffel, van Dooren, Greer \& Mukhopadhyay, 2010). Asimismo, la literatura advierte sobre las limitaciones que este tipo de problemas puede presentar para alcanzar los múltiples requerimientos de las matemáticas escolares.

En los profesores analizados en este artículo, los enunciados verbales (rutinarios) parecen ser una de las principales maneras (quizás la única) de establecer relaciones entre las matemáticas y la cotidianidad de los estudiantes. Este tipo de enunciados puede alimentar, de alguna forma, cierta tradición escolar que desconoce los diferentes roles de las matemáticas en contextos sociales y culturales, y del papel de tales contextos en su aprendizaje (Villa-Ochoa \& Berrío, 2015).

Si bien para los casos implicados en este estudio, más allá de los enunciados verbales, puede haber otras situaciones susceptibles de ser modeladas en el aula de clase, también es cierto que los enunciados verbales (rutinarios) adquieren el rol principal para justificar las relaciones entre las matemáticas escolares y la realidad.

Conforme se ha argumentado en este artículo, la relevancia dada por los profesores a los enunciados verbales (rutinarios) parece justificarse en ciertas limitaciones impuestas a algunas actitudes de los estudiantes que están inmersos en tradiciones "facilistas" de las matemáticas, y en las limitaciones que los currículos rígidos pueden ofrecer. Sin embargo, más allá de estos dos factores, este estudio también muestra que las concepciones de la modelación como la producción de modelos, de los modelos como representaciones simbólicas y de la "realidad" como cualquier tipo de enunciado descrito verbalmente, también se convierten en otros de los argumentos con los que los profesores justifican la solución de problemas rutinarios verbales como la principal manera de hacer modelación en el aula de clase. En otras palabras, al estar la noción básica de modelación relacionada con el estudio de fenómenos de la "realidad", los profesores sienten que, de alguna manera, están trabajando modelación al resolver problemas rutinarios presentados verbalmente, pues para ellos, estas (rutinarias) descripciones textuales "pueden ocurrir" y, en consecuencia, pueden adquirir el estatus de "realidad".

Sea para evitar a los estudiantes la complejidad que existe en otras maneras de hacer modelación o para mostrar la "aplicación" de las matemáticas ya estudiadas, los enunciados verbales (rutinarios) se convierten en uno de los mecanismos más recurrentes para los profesores que intervinieron en este estudio. Estos resultados son coherentes con los reportados en la literatura internacional. Por ejemplo, Cinzia Bonotto (2007) señala que, los word problems son con frecuencia el único medio para proveer en los estudiantes experiencias de matematización y modelación. De este mismo modo, esta investigadora puntualiza las limitaciones que tiene centrarse solo en este tipo de enunciados pues, según ella, al basar las prácticas matemáticas escolares solo en la resolución de word problems se puede promover en los estudiantes la exclusión de consideraciones realistas y una limitación en la construción de sentido, hecho que ya se ha mencionado en este artículo al retomar los planteamientos de Susan Gerofsky (2010) y Lieven Verschaffel, Wim van Dooren, Brian Greer y Swapna Mukhopadhyay (2010).

Conforme los resultados de este estudio muestran, para algunos profesores los problemas presentados en enunciados verbales rutinarios se convierten en la manera privilegiada de hacer modelación matemática escolar. En ese sentido, con base en la literatura internacional (Bonotto, 2007; Gerofsky, 2010), es necesario trascender la idea de los enunciados verbales rutinarios, puesto que estos enunciados tienen un carácter generalmente simplificado y no invitan al estudiante a asumir una actitud activa frente al problema. En otras palabras, es necesario contribuir para que este tipo de problemas vayan más allá de tareas "revestidas" en un contexto real y que en su solución los estudiantes requieren básicamente "desvestirlas" (Palm, 2009). 
Los resultados de este estudio sugieren la necesidad de proporcionar espacios en los cuales los profesores puedan reconocer otras potencialidades de los contextos socioculturales que se han reportado en la literatura, por ejemplo, para producir conocimiento matemático que los estudiantes no hayan estudiado previamente en sus clases de matemáticas. Se hace importante discutir que al limitar el papel de los contextos "reales" a solo aplicaciones, el estudiante está sometido a cumplir un "papel pasivo" de las matemáticas, lo cual niega al estudiante la posibilidad de participar de su producción en el contexto escolar; contrario a ello, se queda en la reproducción de los temas ya presentados por el profesor en la clase como ha sido cuestionado por Jhony Alexander Villa-Ochoa y Carlos Mario Jaramillo-López (2011).

\section{Conclusiones}

En la primera parte de este artículo se mostró que la literatura internacional viene demandando mayores y mejores maneras de integrar la modelación matemática en el día a día de las aulas de clase; asimismo, se describieron algunos aspectos que la investigación internacional ha reportado sobre los elementos que han intervenido en la apropiación y el convencimiento que los profesores tienen de este proceso en las aulas de clase.

Adicional a los aspectos encontrados en la literatura, los resultados de este estudio dan cuenta de que hay profesores para quienes es importante que los estudiantes puedan trascender los conocimientos matemáticos a situaciones de la "realidad"; a pesar de ello, esta "realidad" parece obviar aspectos relativos a los contextos propios de la cultura de los estudiantes, para agotarse en un conjunto de enunciados verbales rutinarios que, a partir de palabras, revisten un contenido matemático en contextos "artificiales" que intentan simplificar la complejidad de tales contextos.

Conforme fue evidenciado en este estudio para algunos profesores los problemas "reales" cumplen un papel de aplicación en las aulas de clase; a pesar de ello, consideran que para que el estudiante pueda tener "éxito" en tales problemas debe estar "dotado", previamente, de habilidades y conocimientos matemáticos, con lo cual descuidan que la modelación matemática es uno de los componentes de la actividad matemática misma y, por tanto, se requiere propiciar espacios de formación en los cuales los estudiantes y profesores puedan afrontar este tipo de componentes.

Los casos reportados en este artículo se convierten en evidencia de que también en el contexto colombiano los enunciados verbales rutinarios parecen ser una herramienta privilegiada para develar "las relaciones" entre las matemáticas escolares y los contextos propios de la cultura. Las evidencias aportadas en este artículo muestran que, para algunos profesores, los problemas rutinarios presentados en enunciados verbales se justifican en la necesidad de "desarrollar la habilidad" para establecer traducciones entre el lenguaje natural y el lenguaje simbólico-matemático; con este único propósito y bajo esta lógica, la mirada se pone en la "traducción" entre lenguajes y, por tanto, los problemas de palabra rutinarios parecen satisfacer las demandas impuestas por los ejercicios prototipo que se privilegian en los espacios escolares.

Aunque la literatura ha reportado significativas críticas frente al papel de los enunciados verbales, la intención de este artículo no es apoyarlas ni contrariarlas, sino cuestionar el reduccionismo al que puede forzarse la modelación matemática cuando se restringe solo a la construcción de una representación simbólica para este tipo de enunciados. Cuando esto ocurre, se corre el riesgo de no atender a las diferentes ventajas que se han reportado en la literatura sobre la modelación matemática (Bassanezi, 2002; Blum \& Borromeo-Ferri, 2009; Villa-Ochoa \& Jaramillo-López, 2011; etc.); de igual manera, como lo señalan Lieven Verschaffel, Wim van Dooren, Brian Greer y Swapna Mukhopadhyay (2010), puede desconocerse la posibilidad de pensar sobre la naturaleza de la modelación en sí misma y sus implicaciones humanas y sociales.

Los resultados de este estudio permiten develar algunas razones en las que se basan los profesores para reducir la modelación matemática escolar a la resolución de problemas rutinarios. Con base en esta comprensión, se derivan algunas implicaciones para la formación inicial y continuada de profesores; de manera particular, se hace necesario promover espacios para la discusión y reflexión sobre la pertinencia de implementar la modelación matemática en las aulas escolares, así como las diversas maneras que pueden identificarse para esta implementación. Este tipo de reflexiones han de tener en cuenta los conocimientos y creencias de los profesores sobre las matemáticas y su enseñanza, según lo han señalado Gabriele Kaiser y Katja Maaß (2007); pero también, sus consideraciones e ideas sobre la modelación matemática y los factores asociados a ella. Es de particular importancia comprender la modelación matemática como un componente de las matemáticas mismas; es decir, como un proceso inherente a la producción matemática y que, como tal, debe ser desarrollado en el aula de clase. De esa forma, se espera no supeditar la modelación matemática ni los contextos socioculturales de los cuales emerge a una mirada un poco "asistencialista o utilitarista" circunscrita solo a una estrategia con fines ilustrativos, de aplicaciones matemáticas o motivacionales en la introducción de un tema matemático; sino que, más allá de ello, se trata 
de reconocer sus potencialidades como referentes para la construcción de conocimiento matemático en relación con la cultura (Villa-Ochoa \& Berrío, 2015). Todo ello implica ver las matemáticas inmersas en determinados contextos socioculturales, y al profesor como un agente que (re)constituye el currículo en términos de las decisiones sobre los tipos de experiencias matemáticas que los estudiantes pueden tener en las aulas de clase.

Si bien hay diversas maneras de articular procesos de modelación en las aulas de clase (Villa-Ochoa, 2013), también es cierto que todas ellas imponen ciertas demandas en los currículos escolares. El caso de los enunciados verbales puede convertirse en una manera de implementar modelación especialmente favorable para aquellos currículos con ciertas características rígidas; sin embargo, es necesario trascender la visión rutinaria de este tipo de problemas para convertirlos en problemas que, aunque cortos, sean realistas o auténticos (Bonotto, 2007).

Lo anterior implica desarrollar estrategias por medio de las cuales los estudiantes puedan ser partícipes de la construcción de tales enunciados, a partir de sus contextos culturales; y si bien, los enunciados verbales son simplificados, los estudiantes pueden ser partícipes de la decisión frente a tales simplificaciones. Sin embargo, sea cual sea la aproximación realizada en el aula de clase, en este caso con los enunciados verbales realistas, se hace necesario valorar las limitaciones en las que se incurra sin pretender que estas miradas se conviertan en estereotipos que agotan la complejidad de la modelación matemática.

Finalmente, los resultados presentados en este estudio reportan necesidades adicionales a las evidenciadas por Mogens Niss (2001) y Cinzia Bonotto (2007); particularmente, la creación de ambientes o comunidades en los que los profesores se empoderen de acciones que discutan los currículos y que les permitan romper con la aparente "rigidez" de los planes basados en las construcciones aisladas de las situaciones que rodean a los estudiantes en su vida cotidiana. En consecuencia, futuras investigaciones pueden enfocarse en el diseño e implementación de tales ambientes y comunidades en los que también se favorezcan la reflexión y el diálogo de experiencias, y que permitan la consolidación de una visión más amplia de lo que puede ser la "realidad", de manera que cobije los contextos sociales y cuturales en los cuales las matemáticas escolares pueden circunscribirse. Como futuras líneas de acción, los programas de formación de profesores podrían discutir y atender a las diversas necesidades evidenciadas en la literatura internacional sobre la modelación matemática y la manera en que se constituye e implementa en las aulas de clase.

\section{Agradecimientos}

Deseo agradecer al Comité para el Desarrollo de la Investigación (CODI) y a la Dirección de Regionalización de la Universidad de Antioquia por la financiación del proyecto del cual se derivó este artículo. Así mismo, quiero agradecer a Yadira Marcela Mesa y a Lina María Muñoz, de la Red Colombiana de Modelación en Educación Matemática, RECOMEM, por los comentarios y críticas realizadas a las diferentes versiones de este documento.

\section{Sobre el autor}

Jhony Alexander Villa-Ochoa es coordinador de la Red Colombiana de Modelación en Educación Matemática, RECOMEM. Profesor del departamento de Enseñanza de las Ciencias y las Artes, Facultad de Educación, Universidad de Antioquia. 


\section{Bibliografía}

Agudelo-Valderrama, C. (2006). The Growing Gap between Colombian Education Policy, Official Claims and Classroom Realities: Insights from Mathematics Teachers' Conceptions of Beginning Algebra and its Teaching Purpose. International Journal of Science and Mathematics Education, 4 (3), 513-544. Disponible en: http:// www.researchgate.net/publication/226247484_The_growing_gap_ between_colombian_education_policy_official_claims_and_classroom realities_insights_from_mathematics_teachers'_conceptions_of_ beginning_algebra_and_its_teaching_purpose

Alsina, C. (2007). Si Enrique VIII tuvo 6 esposas, ¿cuántas tuvo Enrique IV? El realismo en educación matemática y sus implicaciones docentes. Revista Iberoamericana de Educación, 43, 85-101. Disponible en: http://www.rieoei.org/rie43a04.pdf

Araújo, J. (2009). Uma abordagem sócio-crítica da modelagem matemática: a perspectiva da educação matemática crítica. Alexandria, Revista de Educação em Ciência e Tecnologia, 2 (2), 55-68. http://www.mat. ufmg.br/ jussara/artigos.html, https://periodicos.ufsc.br/index.php/ alexandria/article/view/37948/28976

Bassanezi, R. (2002). Ensino-aprendizagem com modelagem matemática. São Paulo: Contexto.

Blum, W. \& Borromeo-Ferri, R. (2009). Mathematical Modelling: Can It Be Taught And Learnt? Journal of Mathematical Modelling and Application, 1 (1), 45-58. Disponible en: http://proxy.furb.br/ojs/index.php/ modelling/article/view/1620/1087

Bonotto, C. (2007). How to Replace Word Problem with Activities of Realistic Mathematical Modelling. In Werner Blum, Peter L. Galbraith, Hans-Wolfgang Henn \& Mogens Niss (eds.). Modelling and Application in Mathematics Educations. The $14^{\text {th }}$ ICMI Study [International Commission in Mathematical Instruction], 185-192. New York: Springer.

Colombia, Ministerio de Educación Nacional, MEN (1998). Lineamientos curriculares: matemáticas. Bogotá: Magisterio. Disponible en: http:// www.mineducacion.gov.co/1621/article-89869.html, http://www. mineducacion.gov.co/1621/articles-89869_archivo_pdf9.pdf

Colombia, Ministerio de Educación Nacional, MEN (2006). Estándares básicos de competencias. Bogotá: Magisterio. Disponible en: http:// www.mineducacion.gov.co/1621/w3-article-340021.html, http:// www.mineducacion.gov.co/1621/articles-340021_recurso_1.pdf

García-García, J. J. \& Rentería-Rodríguez, E. (2013). Resolver problemas y modelizar: un modelo de interacción. magis, Revista Internacional de Investigación en Educación, 5 (11), 297-333. Disponible en: http:// www.redalyc.org/articulo.oa?id $=281028437003$

Gerofsky, S. (2010). The Impossibility of 'Real-Life' Word Problems (according to Bakhtin, Lacan, Zizek and Baudrillard). Discourse: Studies in the Cultural Politics of Education, 31 (1), 61-73. Disponible en: http:// keats.kcl.ac.uk/pluginfile.php/700335/mod_resource/content/1/ Gerofsky\%20\%282010\%291.pdf

Kaiser, G. \& Maaß, K. (2007). Modelling in Lower Secondary Classrooms - Problems and Chances. En Werner Blum, Peter L. Galbraith, HansWolfgang Henn \& Mogens Niss (eds.). Modelling and Application in Mathematics Educations. The $14^{\text {th }}$ ICMI Study [International Commission in Mathematical Instruction], 99-108. New York: Springer. 
Niss, M. (2001). Issues and Problems of Research on the Teaching and Learning of Applications and Modelling. En João Filipe Matos, Werner Blum, Ken Houston \& Susana Paula Carreira (eds.). Modelling and Mathematics Education. International Conference on the Teaching of Mathematical Modelling and Applications, ICTMA 9: Applications in Science and Technology, 72-89. Chichester: Horwood Publishing.

Palm, T. (2009). Theory of Authentic Task Situations. En Lieven Verschaffel, Brian Greer, Wim van Dooren \& Swapna Mukhopadhyay (eds.). Words and Worlds. Modelling Verbal Descriptions of Situations, 3-19. Rotterdam: Sense Publishers. Disponible en: https://www.sensepublishers.com/ media/1281-words-and-worlds.pdf

Rosa, M. \& Orey, D. (2012). A Modelagem como um Ambiente de Aprendizagem para a Conversão do Conhecimento Matemático. Bolema, Boletim de EducaçãoMatemática,26(42A), 261-290. Disponible en: http://www.scielo.br/scielo.php?pid=S0103636X2012000100012\&script=sci_arttext

Schoenfeld, A. H. (1991). On Mathematics as SenseMaking: Informal Attack Unfortunate Divorce of Formal and Informal Mathematics. En James F. Voss, David N. Perkins \& Judith W. Segal (eds.). Informal Reasoning and Education, 311-343. Hillsdale, New Jersey: Erlbaum.

Verschaffel, L. D.; Corte, E. de \& Borghart, I. (1997). Pre-Service Teachers' Conceptions and Beliefs about the Role of Real-World Knowledge in Mathematical Modelling of School Word Problems. Learning and Instruction, 7 (4), 339-359.

Verschaffel, L.; Dooren, W. van; Greer, B. \& Mukhopadhyay, S. (2010). Reconceptualising Word Problems as Exercises in Mathematical Modelling. Journal für Mathematik-Didaktik, 31 (1), 9-29. Disponible en: http://link.springer.com/article/ 10.1007\%2Fs13138-010-0007-x\#page-1

Villa-Ochoa, J. A. (2013). Miradas y actuaciones sobre la modelación matemática en el aula de clase. En Vanilde Bisognin \& Marilaine de Fraga Sant'Ana (eds.). Anais da VIII Conferência Nacional sobre Modelagem Matemática na Educação Matemática, 1-8. Santa Maria, Rio Grande do Sul, Brasil: Centro Universitario Franciscano. Disponible en: http://funes.uniandes.edu.co/2080/1/MC_12 Jhony_Ochoa.pdf

Villa-Ochoa, J., \& Berrío, M. J. (2015). Mathematical Modelling and Culture: An Empirical Study. In G. A. Stillman, W. Blum, \& M. Salett Biembengut (Eds.), Mathematical Modelling in Education Research and Practice, 241-250. Switzerland: Springer International Publishing.
Villa-Ochoa, J. A.; Bustamante, C. A.; Berrío, M.; Osorio, J. A. \& Ocampo, D. A. (2009). Sentido de realidad y modelación matemática. El caso de Alberto. Alexandria, Revista de Educação em Ciência e Tecnologia, 2 (2), 159-180. Disponible en: http://funes.uniandes.edu.co/890/, http://funes. uniandes.edu.co/890/1/jhony.pdf

Villa-Ochoa, J. A. \& Jaramillo-López, C. M. (2011). Sense of Reality through Mathematical Modeling. En Gabriele Kaiser, Werner Blum, Rita BorromeoFerri \& Gloria Stillman (eds.). Trends in the Teaching and Learning of Mathematical Modelling, International Conference on the Teaching of Mathematical Modelling and Applications, ICTMA 14, 701-711. New York: Springer.

Villa-Ochoa, J. A. \& Ruiz-Vahos, H. M. (2009). Modelación en educación matemática: una mirada desde los lineamientos y estándares curriculares colombianos. Revista Virtual Universidad Católica del Norte, 27, 1-21. Disponible en: http:// www.redalyc.org/articulo.oa?id=194215432007

Yin, R. (2009). Case Study Research: Design and Methods. Thousand Oaks, California: Sage Publications. 